\title{
Authors' Index
}

$\mathrm{A}=$ Abstract

A

Abouna, G. 171 (A)

Adelson, J. W. 338 (A)

Affolter, H. 311

Afifi, F. 171 (A), 175 (A), 340 (A)

Afroudakis, A. 203 (A)

Ajdukiewicz, A. 208 (A)

Alcindor, G. L. 338 (A)

Aldrete, J. A. 237 (A)

Amar Costesec, A. 339 (A)

Amstrong, Sylvia J. 173 (A)

Andersen, H. 176 (A)

Andre, C. 108 (A), 339 (A), 352 (A)

Andre, F. 352 (A)

Andreis, P. G. 172 (A)

Anzini, F. 344 (A)

Apostolov, K. 194 (A)

Armato, U. 172 (A), 173 (A)

Armengol, M. J. R. 284

Arondel, J. 189 (A)

B

Bachmann, W. 175 (A)

Bach-Mortensen, N. 214 (A)

Bagshawe, A. F. 174 (A)

Bakken, A. F. 174 (A)

Balabaud, C. 175 (A), 340 (A)

Bali, J. P. 353 (A)

Balmes, J. L. 353 (A)

Barnett, D. 207 (A)

Baron, D. N. 185 (A)

Baron, J. H. 258 (A), 351 (A)

Barros Mott, C. de 359 (A)

Bassetto, M. A. 227 (A)

Bauduin, H. 360 (A)

Bauer, C. 175 (A)

Bauer, D. J. 194 (A)

Beck, I. T. 64

Becker, K. 176(A), 212 (A), 235 (A), 236 (A) 
Beffagna, B. 176 (A)

Bellandi, G. 172 (A)

Bellobuono, A. 176 (A), 206 (A)

Bengmark, S. 180 (A)

Benhamou, J.-P. 175 (A), 188 (A), 340 (A)

Beretta Anguissola, M. 226 (A)

Bernardini, R. 191 (A)

Berona, G. 227 (A)

Bernier, J. J. 341 (A), 354 (A)

Berstad, A. 166 (A)

Berthelot, P. 189 (A), 194 (A), 341 (A),

348 (A) Bertrand, J. L. 342 (A) Bianchi, P. A. 180 (A) Bianchi-Bosisio

Righetti, A. 180 (A) Bianchi Porro, G. 180 (A) Bichel, H. 350 (A) Biernat, J. 1

Billing, H. 208 (A), 221 (A) Binnie, C. 183 (A) Bircher, J. 181 (A) Bird, R. G. 239 (A) Birnbaum, D. 261 Bismuth, H. 194 (A), 348 (A) Bizollon, C. A. 284

Blahnikova, L. 205 (A) Bleichner, G. 230 (A) Bloch, P. 224 (A) Bode, C. 181 (A), 182 (A), 200 (A) Boivin, P. 171 (A), 204 (A) Bonfils, S. 339 (A), 354 (A), 355 (A) Borel, G.-A. 182 (A), 183 (A) Borg, I. 17, 140 Boucherot, Janie 204

(A) Bourel, M. 202 (A), 350 (A) Braito, A. 183 (A) Bralow, S. P. 290 Brandi, D. 230 (A) Brandt, G. 64 Breckenridge, A. 238 (A) Bresadola, F. 191 (A)

Brette, R. 342 (A) Brille, D. 243 (A)

Authors' Index

371

Bringolf, M. 183 (A) Brown, R. 183 (A) Brunt, P. W. 184 (A) Brunner, G. 184 (A), 242 (A) Buchwald, B. 200 (A) Buttery, J. E. 185 (A)

$\mathrm{C}$

Cahlin, E. 235 (A)

Cain, G. D. 209 (A)

Cameron, H. M. 174 (A)

Capurso, P. 344 (A)

Carella, M. 217 (A)

Caroli, J. 185 (A), 208 (A), 213 (A),

(A), 243 (A), 338 (A), 356 (A) Cartei, G. 186 (A), 187 (A) Casini, M. C. 197

(A) Cattoli, G. 180 (A) Cellerino, R. 226 (A), 227 (A) Cerlek, S. 187 (A)

Charbon, G. A. 275 Charbonnier, A. 188 (A) Chattas, A. 355 (A)

Chenderovitch, J. 343 (A) Cherret, A. M. 339 (A) Chiaramonte, M. 193 (A)

Chiarantini, C 197 (A) Chiesara, E. 242 (A) Christoffersen, P. 223 (A) Clark,

C. 183 (A) Clausen, E. 234 (A) Clemente, F. 343 (A) Cohen, R. D. 123 (A),

207 (A) Colomb, E. 343 (A)

COMPERNOLLE, F. 194 (A)

Conard, J. 224 (A)

Corazziari, E. 344 (A)

Corrocher, R. 227 (A)

Costa, G. 171 (A)

Costantino, D. 180 (A), 190 (A), 221 
Cowley, D. J. 351 (A)

Crassous, J. 194 (A), 348 (A)

Creutzfeldt, W. 219 (A), 242 (A)

Cros, R. C. 196 (A)

Crotte, C. 199 (A)

Curry, S. 217 (A)

D

Dam, J. 241 (A)

Damgaard, S. 238 (A)

Dane, D. S. 190 (A)

D'Angelo, A. 222 (A)

Davenport, H. W. 162

Dawson, A. W. 317

Debray, Ch. 345 (A)

De Carlo, P. 197 (A)

Decker, K. 211 (A)

de Franchis, R. 190 (A), 206 (A), 207 (A)

Degos, F. 230 (A)

Degos, J.-D. 230 (A)

De Groote, J. F. 194 (A)

Deicher, H. 221 (A)

Del Nrnno, E. 206 (A), 207 (A)

Del Prete, S. 180 (A), 221 (A)

de Saint Laurent, J. E. 185 (A)

Descos, F. 339 (A), 352 (A)

Desjeux, J.-F. 188 (A)

Desmet, V. J. 189 (A)

Desoize, B. 339 (A)

Desvigne, C. 284

De Wolf-Peeters, C. 189 (A)

DeVos, R. 189(A)

Dhumeaux, D. 188 (A), 189 (A), 341 (A)

Diallo, T. 182 (A)

Dickenson, G. 209 (A)

Dickson, G. H. 49

Dietrichson, O. 223 (A)

Dini, M. 204 (A)

Dioguardi, N. 190 (A), 206 (A), 207 (A),

326 Dive, C. 316

Doglia, M. 180 (A), 221 (A) Dölle, W. 191 (A), 198 (A) Doniach, D. 190 (A)

Donini, I. 191 (A) Doss, M. 191 (A) Dowling, R. H. 202 (A) Dubrasquet, M.

355 (A) Duclert, N. 345 (A) Dudley, F. J. 192 (A), 194 (A) Duffaut, M. 346 (A)

Duhurt, J. 224 (A) Dumont, M. 188 (A), 193 (A), 347 (A) Dura, J. 227 (A)

372

Authors' Index 
Dyer, N. H. 317

E

Eccher, C. 173 (A)

Efstratopoulos, A. 203 (A)

Eisenburg, J. 236 (A)

Elling, P. 223 (A)

Eloy, M. R. 358 (A)

Emås, S. 17, 140

Erb, W. 192 (A)

Erlinger, S. 188 (A), 193 (A), 347 (A)

Evrard, E. 108

$\mathrm{F}$

Faarup, P. 210 (A)

Fagiolo, U. 193 (A)

Farina, L. 176 (A), 196 (A)

Farini, R. 193 (A), 222 (A)

Farrow, L. J. 239 (A)

Fassati, L. R. 176 (A), 196 (A)

Faudemay, F. 357 (A)

Fauvert, R. 171 (A)

Feldmann, G. 194 (A), 204 (A), 348 (A)

Ferrand, B. 350 (A)

Fevery, J. 194 (A)

Figarella, C. 343 (A)

Filosa, E. 207 (A)

Fisher, L. 171 (A)

Fleischmann, R. A. 237 (A)

Flute, P. T. 230 (A)

Fox, R. A. 192 (A), 194 (A)

Franco, D. 194 (A), 348 (A)

Francois, B. 342 (A)

Frei, J. 223 (A)

Freixinos, J. 349 (A)

Frezza, M. 226 (A), 227 (A)

Fric, P. 40

Frommer, D. 195 (A)

Fukui, I. 153

G

Galmarini, D. 176 (A), 196 (A) Gallot, D. 224 (A) Gauthier, A. P. 196 (A)

Gelis, Ch. 350 (A) Gentilini, P. 197 (A), 198 (A) Gerard, P. 225 (A)

Gerdes, H. 198 (A)

Gerhard, L. 198 (A)

Gerolami, A. 199 (A)

Ghidoni, A. 176 (A)

Gianotti, F. 200 (A), 221 (A)

Gillet, M. 358 (A) 
Ginestal da Cruz, A. 228 (A)

Goebell, H. 181 (A), 182 (A), 200 (A)

Golding, P. L. 200 (A)

Grabner, G. 205 (A)

Graef, J. 352 (A)

Grainger, J. M. 201 (A)

Granger, J. 213 (A), 224 (A)

Grenier, J. F. 358 (A)

Greten, H. 201 (A), 237 (A)

Gribomont, B. 225 (A)

Grob, P. J. 201 (A)

Groen, J. H. 261

Grün, M. 213 (A)

Gudmand-Höyer, E. 316

Gueguen, C. 202 (A), 350 (A)

Guibout, P. 204 (A)

Guillouzo, A. 202 (A), 350 (A)

Gullo, L. 259 (A)

Gunawardene, K. R. W. 9

Gupta, M. C. 202 (A)

Gustafsson, A. 235 (A)

Gustosi, A. 203 (A)

$\mathrm{H}$

Hachelnoffer, J. 358 (A)

Häcki, W. 181 (A)

Hadchouel, P. 185 (A), 224 (A), 243 (A)

Hadorn, B. 355 (A)

Hadziyannis, S. 203 (A)

Hakim, J. 204 (A)

Halpern, A. 181 (A)

Hansky, J. 351 (A)

Harry, D. S. 204 (A)

Hatzfeld, 243 (A)

Haykawa, H. 127

Heap, R. C. 216 (A)

Hecht, Y. 213 (A)

Heirwegh, K. P. M. 194 (A)

Heissmeyer, H. 239 (A)

Henning, H. 191 (A)

Higurachi, H. 127

Authors' Index

373

Hillenbrand, P. 208 (A) Hirayama, C. 31 Hoensch, H. 58 Hörder, M. H. 213 (A) Holborow, E. J. 239 (A) Holle, G. 232 (A) Holm-Nielsen, A. 210 (A) 
Horak, W. 205 (A) Horky, J. 205 (A) Huchzermeyer, H. 198 (A) Huguet, C. 224 (A) Hume, D. M. 171 (A) Hunter, J. 206 (A)

I

Ichikawa, H. 127 Ideo, G. 206 (A), 207 (A) 326 Infante, R. 208 (A), 355 (A)

Iles, R. A. 207 (A) Infante, R. 338 (A), 355 (A) Izzo, E. 234 (A)

$\mathrm{J}$

Jacini, A. 221 (A)

Jahn, H. 240 (A)

James, I. M. 216 (A), 244 (A)

Jannuzzi, C. 240 (A)

Jansen, F. H. 208 (A), 221 (A)

Jedrychowski, A. 208 (A)

Jemelka, H. J. 201 (A)

J日DL, J. 40

Jönsson, J. 235 (A) Johansen, S. 222 (A) Jones, D. 183 (A), 209 (A) Jones, E.

A. 209 (A) Jørgensen, M. 209 (A), 210 (A)

$\mathrm{K}$

Kagami, H. 215 (A) Kalden, J. 221 (A) Kaplan, M. M. 180 (A) Karamountzos, D. 203 (A) Karsai, T. 253 Kastanakis, S. 211 (A) Kato, A. 167 (A) Kautzsch, E. F. 210 (A) Kawabe, M. 31

Kayser, W. 232 (A) Kehayoglou, K. 211 (A) Kemp, A. 200 (A) Kennedy, B. J. 125 (A) Keppler, D. 211 (A) Kestens, P. J. 213 (A), 225 (A) Kew, M. 184 (A)

Kimura, N. 31 Kirsten, H. 232 (A) Klapdor, R. 176 (A) Knöbel, H. 212(A)

Kössling, F. K. 218 (A) Komeda, T. 153 Kono, T. 153

KONTUREK, S. J. 1

Koudahl, G. 238 (A) Koumanov, K. 208 (A) Korman, M. G. 351 (A) Koza, J. 227 (A) Krassnitzky, O. 227 (A) Kreel, L. 212 (A) Kreis, B. 204 (A) Kulich, V. 227 (A) Kunakura, K. 127 Kunz, H. 64

$\mathrm{L}$

Lambert, R. 100, 116, 339 (A), 352 (A)

Lambotte, L. 213 (A), 225 (A)

Laor, J. 65

Larsen, H. R. 234 (A)

Laval, J. 346 (A)

Lebenthal, E. 65

Lebert, A. 341 (A)

Lebrec, D. 354 (A)

LeDonne, C. 284

Le Guilly, Y. 202 (A), 350 (A)

Leynweber, W. 192 (A)

Lemp, R. 182 (A)

Lenoir, P. 202 (A), 350 (A)

Lenriot, J. P. 215 (A)

Lesch, R. 175 (A), 239 (A)

Levy, V. G. 213 (A), 243 (A)

Lewitus, Z. 65 
Liehr, H. 213 (A)

Limbosch, J. M. 352 (A)

Lindskov, J. 214 (A)

374

Authors' Index

Lipshutz, W. 123 (A) Littmann, K.-P. 198 (A) Loisance, D. Y. 215 (A) Lojda, Z. 40 Look, D. 191 (A) Louis, A. 346 (A)

$\mathrm{M}$

MacDonell, L. 216 (A)

Machtey, I. 65

Maillard, J.-N. 216 (A)

Magnenat, P. 182 (A), 183 (A), 223 (A)

Malamos, B. 211 (A)

Malis, F. 40

Marleau, D. 230 (A)

Martin, F. 353 (A)

Maschio, G. 222 (A)

Masi, R. 198 (A)

Maxwell, J. D. 206 (A), 217 (A)

Mary, J. Y. 354 (A)

Mbendi, S. 352 (A)

McIntyre, N. 204 (A)

Meneses, L. M. 228 (A)

Merikas, G. 203 (A)

Mersch-Baumert, K. 213 (A)

Meuwissen, J. A. T. P. 217 (A)

Meyer zum Büschefelde, K. H. 218 (A).

219 (A) Michel, H. 219 (A), 230 (A) Mignon, M. 354 (A) Mikulecky, M. 219

(A) Milhaud, G. 185 (A) Miller, J. R. M. 174 (A) Mischer, P. A. 218 (A)

Mitchell, C. 200 (A) Modigliani, M. 354 (A) Montemagno, M. 196 (A)

Morganti, A. 326 Morisot, P. 224 (A) Moroni, G. A. 221 (A) Morotomi, I. 31

Moussouros, A. 203 (A) Mózsik, Gy. 253 Mule, A. 199 (A) Müller, M. 244 (A)

Müller, R. 221 (A) Murakami, T. 127

Murphy, G. M. 221 (A) Fr. Myren, J. 222 (A)

$\mathrm{N}$

Näccarato, R. 186 (A), 187 (A), 193 (A), 223 (A) Nagaratnam, N. 9 Nawrocki, P. 191 (A) Neale, G. 202 (A), 238 (A)

Nepveux, P. 188 (A) Neuberger, H. O. 182 (A) Nicholls, R. J. 223 (A) Nielsen, J. O. 223 (A) Nilsson, S. 235 (A) Nishizawa, M. 127 Nissen, R. 64 Norby

Rasmussen, S. 231 (A)

NORGAARD, T. 210 (A)

o

Oetliker, O. 355 (A)

Okolicsanyi, L. 223 (A)

Okubo, K. 153 
Oldershausen, H. F. v. 237 (A)

Olsen, C. 224 (A)

Olsson, R. 180 (A)

Opolon, P. 224 (A)

Orme, M. 238 (A)

Ossi, E. 222 (A)

Otte, J. B. 213 (A), 225 (A)

Owens, D. 225 (A)

$\mathrm{P}$

Pannell, G. 230 (A) Panzetta, O. 187 (A) Papacostas, D. 211 (A) Papenberg, J.

226 (A) Paran, M. 219 (A), 350 (A) Parbhoo, S. P. 185 (A), 208 (A) Parkash,

Om 269 Parkes, D. 217 (A) Paumgartner, G. 205 (A) Pelizzo, M. R. 242 (A)

Penaud, J. 194 (A), 348 (A) Pensendorfer, F. 227 (A) Pequignot, E. 357 (A)

Authors' Index

375

Perings, E. 184 (A), 219 (A), 242 (A)

Perona, G. 226 (A)

Peronneau, P. 215 (A)

Persson, B. 235 (A)

Petera, V. 227 (A)

Peters, C. M. 129

Petersen, H. 166 (A)

Pfeiffer, C. J. 129

Picao F. J. 228 (A)

Pieroni, C. 198 (A)

Pinto Correia, J. 228 (A)

Pisani, F. 196 (A)

Pirk, F. 89

Pointard, L. 175 (A), 340 (A)

Pointner, H. 355 (A)

Potet, F. 345 (A)

Preaux, A.-M. 189 (A), 194 (A), 348 (A)

Preisig, R. 181 (A)

Prioton, J.-B. 230 (A)

Pucci, C. 196 (A)

Pujol, J.-P. 230 (A)

Puricelli, C. 196 (A)

$\mathrm{R}$

Radecki, T. 1.

Rake, M. O. 230 (A)

Ranek, L. 231 (A), 234 (A)

Reerink, E. 232 (A)

Remmer, H. 237 (A)

Renaud, G. 356 (A)

Renger, F. 232 (A) 
Rettenmaier, G. 233 (A)

Reutter, W. 175 (A)

Rey, J. 356 (A)

Reynard, A. P. 233 (A)

Ribeiro, T. 343 (A)

Ribet, A. 346 (A), 349 (A)

Richardson, A. 206 (A)

Ricour, C. 356 (A)

Rob, C. 252 (B)

Robinson, J. 206 (A)

Roche, J. 230 (A)

Rösler, H. 181 (A)

Romieu, C. 219 (A), 237 (A), 350 (A)

Roschlau, G. 232 (A)

Ross, A. 234 (A)

Roze, Cl. 345 (A)

Rozen, R. 357 (A)

Rudigier, J. 211 (A)

Rüedi, B. 183 (A)

Rueff, B. 204 (A), 216 (A), 230 (A)

Ruggiero, G. 234 (A)

$\mathrm{S}$

Salvadori, G. 197 (A), 198 (A)

Sandhop, W. 235 (A)

Saracbasi, Z. 241 (A)

Sarles, H. 196 (A), 199 (A), 343 (A),

359 (A) Sausse, F. 213 (A), 224 (A) Sautin, Cl. 357 (A) Schersten, T. 235 (A)

Scheurer, P. J. 184 (A), 201 (A) Schlamp, R. 236 (A) Schmid, M. 201 (A)

Schmid, R. 174 (A) Schmidt, A. 191 (A) Schmidt, M. 236 (A) Schmidt, V. 233

(A) Schoene, B. 237 (A) Schreiber, G. 244 (A) Schultz, R. 237 (A) Schultz, S.

M. 355 (A) Schulenberg, P. F. 232 (A) Seebach, H. B. von 243 (A) Seidel, D.

201 (A), 237 (A) Selway, J. W. 194 (A) Serrou, B. 219 (A), 237 (A), 350 (A)

Sestoft, L. 238 (A) Sherlock, S. 184 (A), 192 (A), 194 (A),

195 (A), 208 (A), 234 (A), 244 (A) Shiner, M. 69 Shirakabe, H. 127 Shmerling,

D. H. 69 Sicot, C. 216 (A), 230 (A) Silver, J. 238 (A) Sinclair, D. G. 64

Simpson, B. R. 207 (A) Sivacki, J. 187 (A) Skala, I. 89

Smith, M. 183 (A), 200 (A) Smith, R. 253 Solassol, C. 219 (A), 350 (A)

376

Authors' Index

Soler, Ch. 338 (A) Soler-Argilaga, C. 356 (A) Sonksen, P. 225 (A) Souchard, M. 345 (A) Soussen-Colle, M. C. 352 (A) Srivastava, M. 225 (A) Stewart, D.

A. 206 (A) Stern, A. 224 (A) Stein, H. 261 Stein, U. 239 (A) Stella, G. D. 242

(A) Sterb, A. 213 (A) Stich, W. 236 (A) Stock, Ch. 358 (A) Stoebner, P. 358

(A) Strohmeyer, G. 191 (A) Strunin, J. 207 (A) Strunin, L. 207 (A) Sturniolo,

G. C. 193 (A) Surrenti, C. 197 (A), 198 (A) Svensson, S.-O. 17 
$\mathrm{U}$

Ulagay, I. 241 (A) Urban, J. 244 (A) Urbanke, A. 187 (A) Utili, R. 234 (A)

Vagne, M. 284 Vallo, F. 238 (A) Vansteenkiste, Y. 360 (A) Vassanelli, P. 242 (A) Vatier, J. 354 (A) Vatier, M. 355 (A) Vaysse, N. 346 (A) Vecchietti, M.

176 (A) Vercesi, G. 176 (A), 196 (A) Vesely, V. 227 (A) Vido, I. 184 (A), 242 (A) Vigoni, F. 349 (A) Vincent, D. 360 (A) Vincent, J. 243 (A) Vojtechovsky, M. 205 (A)

Takahata, J. 153 Tassara, A. 183 (A) Tassiopoulos, T. 243 (A) Tarenzi, L. 176 (A) Taylor, P. E. 233 (A), 239 (A) Teichmann, F. 253 Teichmann, W. 240 (A) Teodori, U. 198 (A) Thaler, M. 174 (A) Thambugala, R. L. 358 (A)

Theodoropoulos, G. 185 (A) Thivolet, J. 100 Thompson, A. E. 49 Thompson, G. R. 238 (A) Thor, P. 1

Tiscornia, O. 259 (A) Tolentino, P. 240 (A) Torsoli, A. 344 (A) Tour, J. de la, 345 (A) Trepo, C. 100 Trip, J. A. J. 241 (A) Tursz, T. 204 (A) Tygstrup, N. 234

(A)

W

Walczak, J. 192 (A)

Walsh, J. H. 190 (A)

Weigand, K. 244 (A)

Weiss, Y. 230 (A)

Wengeler, H. 201 (A), 237 (A)

Wewalka, F. 227 (A)

Whelan, G. 244 (A)

Wieland, H. 237 (A)

Willems, G. 360 (A)

Williams, R. 200 (A), 206 (A), 217 (A),

230 (A) Wohlgemuth, B. 232 (A)

$\mathrm{X}$

Xanalatos, C. 216 (A), 244 (A)

Zampieri, G. 221 (A)

Zeitoun, P. 345 (A), 339 (A)

Zelder, O. 182 (A)

Zimmermann, W. 239 (A)

Zuckerman, A. J. 173 (A), 238 (A), 239 (A)

Zurli, J. 196 (A) 